\title{
Outer Leaflet of the Lipid Bilayer
}

National Cancer Institute

\section{Source}

National Cancer Institute. Outer Leaflet of the Lipid Bilayer. NCI Thesaurus. Code C33234.

The extracellular facing lipid layer of the plasma membrane. 